\title{
Entrainment, Rayleigh Friction, and Boundary Layer Winds over the Tropical Pacific
}

\author{
BJORN STEVENS, ${ }^{1}$ JIANJUN DUAN \\ Department of Atmospheric Sciences, \\ University of California Los Angeles, Los Angeles California, USA, \\ JAMES C. MCWILLIAMS, MATTHIAS MÜNNICH AND J. DAVID NEELIN \\ Department of Atmospheric Sciences and Institute for Geo- and Planetary-Physics \\ University of California Los Angeles, Los Angeles California, USA
}

Submitted to the Journal of Climate, January 2001

Revised June 2001

\begin{abstract}
Winds over the tropical Pacific are interpreted using mixed-layer theory. The theory - which posits that the surface winds can be derived in terms of a force balance between surface drag, pressuregradients, Coriolis forces and the vertical mixing of momentum into the boundary layer (entrainment) is very successful in predicting the seasonal climatology of the surface winds. The model is also used as a basis for interpreting previous results. In particular the model illustrates why studies that model the momentum flux divergence as a Rayleigh damping find optimal damping coefficients that are anisotropic. A linear variant of the model, that also incorporates entrainment but neglects the quadratic relation between the wind-speed and the surface stress, is also found to skillfully predict the surface winds. In addition to improving the representation of the winds, it leads to realistic representations of the divergence of the vector wind. If the key parameters of the model (the entrainment rate and the boundary layer depth) are assumed to have uniform climatological mean values over the Pacific basin, optimal parameter values can be derived by matching the model winds to the climatology. Such a procedure leads to boundary layer depths between $300-400 \mathrm{~m}$ and entrainment rates slightly less than $1 \mathrm{~cm} \mathrm{~s}^{-1}$. A somewhat more general model of the boundary layer winds (the so-called $K$-profile parameterization), is used to show that accounting for the vertical structure of the wind-profiles yields somewhat larger optimal estimates of $w_{e}$ and $h$. Overall the incorporation of the entrainment effect is critical, indicating that the acceleration of the near surface winds by momentum mixing with the free atmosphere is a first-order effect that should not be neglected in simple models. Physically this effect is one of resisting the turning of the winds.
\end{abstract}

\footnotetext{
${ }^{1}$ Corresponding Author Address: Bjorn Stevens, Department of Atmospheric Sciences, University of California Los Angeles, 405 Hilgard Ave., Box 951565, Los Angeles, CA 90095-1565. Email: bstevens@ atmos.ucla.edu.
} 


\section{Introduction}

A starting point for many models of the surface (or boundary layer) winds over the tropical oceans is the generalized Ekman balance

$$
f \mathbf{k} \times \overline{\mathbf{u}}+\frac{1}{\rho_{0}} \nabla \bar{p}=\frac{\partial \boldsymbol{\tau}}{\partial z}
$$

where $\rho_{0}$ is the basic state density, $p$ is pressure and $\overline{\mathbf{u}}=(\bar{u}, \bar{v})$, is the expected value of the wind. The balance reflects the assumption that the direct effects of viscosity as well as the effects of departures from horizontal homogeneity or stationarity in the wind field are negligible. Indirectly viscosity manifests itself through the presence of the turbulent stress, $\boldsymbol{\tau}=-\left(\overline{u^{\prime} w^{\prime}}, \overline{v^{\prime} w^{\prime}}\right)$. By integrating (1) over the depth $h$ of the atmospheric boundary layer (ABL), an analogous balance,

$$
f \mathbf{k} \times \mathbf{U}+\frac{1}{\rho_{0}} \nabla P=\frac{\boldsymbol{\tau}(h)-\boldsymbol{\tau}(0)}{h},
$$

can be expressed for the bulk wind,

$$
\mathbf{U}=\frac{1}{h} \int_{0}^{h} \overline{\mathbf{u}} d z
$$

Past work has tended to emphasize a simplified variant of the above balance,

$$
f \mathbf{k} \times \mathbf{U}+\frac{1}{\rho_{0}} \nabla P=-\varepsilon \mathbf{U},
$$

which we call the Rayleigh Friction Model (RFM) because the flux divergence term on the right-hand side of (2) has been replaced by a simple Rayleigh drag. This drag (or friction) mimics the effect of surface fluxes by damping the wind on a specified timescale. Models similar to (3) have a long history in the literature, and although we have formulated the RFM in terms of the bulk wind, the existing literature is ambiguous on this point; some studies invoke Rayleigh damping in terms of (1), thereby allowing for a distinction between the bulk and the surface wind, yet others invoke Rayleigh damping in related but different contexts (e.g., Matsuno, 1966, and many subsequently). However, studies which attempt to physically justify the use of Rayleigh damping in models of the winds typically do so in terms of balance equation for the bulk winds. The justification being that to a first approximation surface drag behaves linearly and $h$ corresponds to a level at which $\tau$ can be neglected, (Lindzen and Nigam, 1987; Neelin, 1988). From the perspective of the climatological wind-balance the latter assumption implies that vertical mixing of momentum into the ABL plays no important role in determining the pattern of surface winds

The popularity of the RFM stems both from its simplicity, and from the perception that it provides a reasonable description of the ABL winds. For example, Murphree and van den Dool (1988) demonstrate that (3), forced by surface pressure gradients, exhibits marked skill in its prediction of the seasonal climatology of surface winds. Their work also indicates that:

"Somewhat better $\bar{u}(\bar{v})$ skills [arise] if a smaller (larger) than standard $\varepsilon$ value is used."

This apparent anisotropy was explored more systematically by Deser (1993). By allowing $\varepsilon$ to take on different values depending on whether one was considering the meridional or zonal momentum balance she proposed a modified version of the RFM

$$
\begin{aligned}
& \frac{1}{\rho_{0}} \frac{\partial P}{\partial x}-f V=-\varepsilon_{x} U \\
& \frac{1}{\rho_{0}} \frac{\partial P}{\partial y}+f U=-\varepsilon_{y} V
\end{aligned}
$$


which we call the anisotropic Rayleigh Friction Model (ARFM) to distinguish it from the isotropic, or rotationally invariant, RFM given by (3). Because different values of $\varepsilon_{x}$ and $\varepsilon_{y}$ have no a priori justification one is forced to estimate their values by fitting ( $4 a)$ and (4b) to the data. Following such a procedure Deser associated $\varepsilon_{x}$ with the slope of the line arising from the regression of the left-hand side of $(4 a)$ onto $\bar{u}$. Using the meridional balance equation $(4 b), \varepsilon_{y}$ was fit following a similar procedure. Result suggest that the ARFM fits the data best if $\varepsilon_{y}$ is two to three times larger than $\varepsilon_{x}$. Subsequent analyses (Li and Wang, 1994; Chiang and Zebiak, 2000) supported the Deser findings although the fitting procedure was shown to have a marked latitudinal dependence - both $\varepsilon_{x}$ and $\varepsilon_{y}$ tend to increase away from the equator.

Why do the derived friction coefficients behave as they do? Deser argued that the differences between $\varepsilon_{x}$ and $\varepsilon_{y}$ reflects an anisotropy in the ABL winds themselves. For instance, during the Atlantic Tradewind Experiment (ATEX e.g., Augstein et al., 1974), the meridional winds were observed to peak near the surface, while the zonal winds have their maximum nearer $950 \mathrm{hPa}$ (Brümmer et al., 1974; Stevens et al., 2001), which is the approximate height of cloud base. Deser speculated that the source of the anisotropy in the ABL winds, was the anisotropy of the free tropospheric wind, which above the ABL tends to be predominantly zonal. These ideas received support from a recent study by Chiang and Zebiak (2000) thus suggesting that an alternative explanation ( $\mathrm{Li}$ and Wang, 1994), which attributes the anisotropy in $\varepsilon_{x}$ and $\varepsilon_{y}$ to horizontal flux convergence associated with transients, is not necessary.

Both the arguments by Deser (1993) and the model of Chiang and Zebiak (2000) take the ABL wind profiles for granted. Which is to say that the wind profiles are not determined by their arguments. As a result their explanations as to the origins of the asymmetry in the friction coefficients, while plausible, are proximate. Moreover, because neither study addresses the theoretical basis for the RFM approximation (Lindzen and Nigam, 1987; Neelin, 1988), neither tackles the apparent paradox whereby the attribution of differences between $\varepsilon_{x}$ and $\varepsilon_{y}$ is to processes which are formally neglected in most attempts to justify RFM models in the first place.

The purpose of this work is to revisit these issues and answer these questions. We frame our study about an examination of the ability of generalized Ekman balances to explain the ABL wind-budget over the tropical oceans. In particular we are interested in understanding and clarifying the role of entrainment in such balances. Our motivation is twofold: First it is clear that in some neighborhood of the equator entrainment must contribute at leading order to balances that resemble (1). Second, we want to clarify the theoretical basis for the ARFM, which, while often justified in reference to entrainment, fails to systematically incorporate this effect. We proceed as follows: in $\S 2$ we introduce the mixed layer model, and show that for uniform parameter values it provides a remarkable description of the surface winds. The explicit inclusion of vertical mixing of momentum into the $\mathrm{ABL}$ is a critical component which both improves the skill of the model and provides a rational basis for interpreting previous results. In $\S 3$ we derive a linearized version of the model which retains the entrainment effect and also demonstrates marked skill. Lastly in $\S 4$ we briefly explore the behavior of a somewhat more complicated model of the ABL wind, one that accounts for its vertical structure. This allows us to explore the physical distinction between the bulk and surface values of the wind and the implication of this distinction on our findings.

\section{Mixed Layer Analysis}

a. A mixed-layer model of the winds

To close (2) requires that we model the boundary fluxes in terms of the bulk wind, $\mathbf{U}$, and other parameters or forcings. Straightforward models for these fluxes can be constructed on the basis of conventional arguments. Following Deardorff (1972) the surface flux $\left(u_{*}^{2} \equiv\|\boldsymbol{\tau}(0)\|\right)$ is applied in the direction of the ABL wind,

$$
\boldsymbol{\tau}(0)=\left(C_{D}\|\mathbf{U}\|\right) \mathbf{U},
$$


where $C_{D}$ is a bulk exchange coefficient. Deardorff develops explicit formulae for $C_{D}$ which depend on the stability, the ABL depth, $h$, and the surface roughness. Over the tropical oceans typical values of these parameters yield $C_{D} \approx 1 / 900$, which is also in accord with the recommendation of Garratt (1992). Modeling the mean drag in terms of the mean wind neglects the effect of fluctuations in the wind, but can be justified if such fluctuations are small, or if they can be absorbed in the specification of $C_{D}$. The latter is not something we attempt to do at this point.

Instead of neglecting entrainment as a source of momentum, we include it using a simple bulk entrainment law (e.g., Lilly, 1968),

$$
\boldsymbol{\tau}(h)=w_{e} \Delta \mathbf{U}=w_{e}\left(\mathbf{U}_{T}-\mathbf{U}\right),
$$

where $w_{e}$ is an entrainment velocity and $\mathbf{U}_{T}$ is the vector wind above the ABL. This sort of relationship is appropriate for profiles wherein the winds are well-mixed throughout the upper portion of the ABL, and then adjust to their free-atmospheric values over a thin layer. In the present context such an approach is motivated by the identification of the sub-cloud layer with the momentum boundary layer (e.g., Brümmer et al., 1974; Stevens et al., 2001); thus, we identify $h$ as the height of cloud base. Although (6) makes no explicit statement about the importance of momentum transport by shallow convection, such processes are indirectly implicated at least to the extent that $w_{e}$ is not equal to the subsidence velocity at $z=h$. In addition, moist convective processes indirectly affect the momentum budget through their regulation of the sub-cloud layer depth.

Given the closure assumptions (interface rules) (5) and (6) we arrive at a bulk wind-law

$$
f \mathbf{k} \times \mathbf{U}+\frac{1}{\rho_{0}} \nabla P=-\mathbf{U}\left(\frac{\|\mathbf{U}\| C_{D}+w_{e}}{h}\right)+\mathbf{U}_{T} \frac{w_{e}}{h},
$$

which we call the mixed layer model (MLM). The MLM has long been used to interpret ABL wind profiles. Our use of it follows standard practices (e.g., Garratt, 1992), except rather than $h$ and $w_{e}$ themselves being determined by the model here they are cast as parameters. The MLM is only slightly more complicated than the Rayleigh friction type models, and for the special case of zero entrainment $\left(w_{e}=0\right)$ and linear drag, it reduces to (3) as expected. Given a specification of the free parameters, and the forcings $\left(\mathbf{U}_{T}\right.$ and $P$ ), a Newton-Raphson procedure converges to 64 bit accuracy in about four iterations. Thus the MLM is also a rather efficient representation of the winds.

Intuition regarding the behavior of (7) can be developed by examining its solutions for idealized flows. For instance a relevant tropical analog is purely zonal free-tropospheric winds balanced geostrophically by meridional pressure gradients. In this case $f U_{T}=-\partial P / \partial y$ and both $V_{T}$ and $\partial P / \partial x$ vanish. For this class of forcing solutions to (7) can be explored as a function of $f$ for varying values of $U_{T}$, or the parameters $h$ and $w_{e}$ (throughout we consider $C_{D}$ to be constant). Results from solutions to (7) for this case are plotted in Fig. 1 for different values of $U_{T}$ (panel a), $h$ (panel b) and $w_{e}$ (panel c).

Because pressure gradients increase away from the equator for the given $U_{T}$ forcing (proportionally to $f$ ) the acceleration of the ABL wind is able to offset surface drag for smaller velocity defects $\left(U_{T}-U\right)$ as $f$ increases, thus $U$ approaches $U_{T}$ at higher latitudes and $V$ is largest in near-equatorial regions where $f$ is non-zero yet the velocity defects are still large. This general behavior is evident in each of the panels of Fig. 1. In panel (a) solutions for different values of $U_{T}$ are rather similar (in a non-dimensional sense) but not exactly so, reflecting the relatively small influence of the non-linearity in the drag term. Increasing $h$ (panel b) has the effect of contracting the equatorial zone which we define as the region where the turning of the wind is most pronounced. Not surprisingly the sensitivity of the model to $w_{e}$ (panel c) is most evident in this zone. This follows because $\left\|\mathbf{U}_{T}-\mathbf{U}\right\|$ is largest and the entrainment flux of momentum is greatest in this region. Overall entrainment tends to reduce the turning of the winds, but the extent of this effect depends on both $f$ and $h$. 

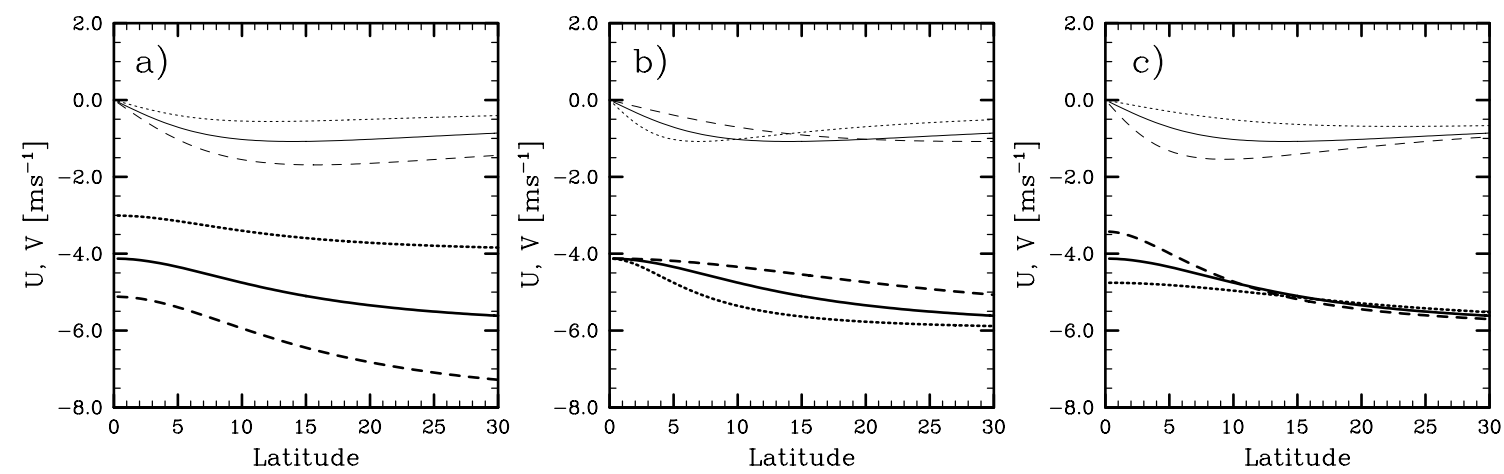

Figure 1: MLM-derived zonal winds (solid thickened lines) and meridional winds (solid thin lines) for $U_{T}=-6$ $\mathrm{ms}^{-1}, h=500 \mathrm{~m}$ and $w_{e}=1 \mathrm{cms}^{-1}$. The solid lines are the same in all panels and should be compared to the dashed/dotted lines which represent changes to the forcings or parameters. (a) Sensitivity to forcing: $U_{T}=-8 \mathrm{~ms}^{-1}$ (dashed) and $-4 \mathrm{~ms}^{-1}$ (dotted). (b) Sensitivity to ABL depth: $h=1000 \mathrm{~m}$ (dotted) and $h=250 \mathrm{~m}$ (dashed). (c) Sensitivity to entrainment: $w_{e}=2 \mathrm{cms}^{-1}$ (dotted) and $w_{e}=0.5 \mathrm{cms}^{-1}$ (dashed).

\section{b. Surface winds}

\section{1) SKILL MEASURES}

To evaluate how well (7) can capture the seasonal climatology of the surface winds we compare results from the model to the climatology over a $1^{\circ}$ grid spanning the the equatorial Pacific basin between $100 \mathrm{~W}-120 \mathrm{E}$, and 20S-20N. At each ocean point on the grid (7) is solved for $\mathbf{U}$, using a fixed value of $\rho_{0}=1.15 \mathrm{gkg}^{-1}$ and the seasonal climatology of $\mathbf{U}_{T}$ and $P$. For $\mathbf{U}_{T}$ we use the $850 \mathrm{hPa}$ winds from the 1979-1995 monthly mean National Centers for Environmental Prediction (NCEP; Kalnay and Coauthors, 1996) reanalysis data set. These data are originally provided on a $2.5^{\circ}$ grid and are bilinearly interpolated to the analysis grid. The $850 \mathrm{hPa}$ winds are used as a proxy for the winds above the top of the momentum boundary layer. While 925 $\mathrm{hPa}$ or $950 \mathrm{hPa}$ winds might be closer to the top of the ABL given values of $h$ we consider, we chose the $850 \mathrm{hPa}$ winds to minimize the effect of the boundary layer scheme used in the context of the reanalysis. Surface pressure gradients estimated from the UWM/COADS 1945-1989 monthly climatology (Da Silva et al., 1994) are used as a proxy for the mean pressure gradient over the entire ABL. Because we expect pressure gradients to be largest at the surface this probably exaggerates the strength of the pressure gradient, although the shallowness of the momentum boundary layer helps mitigate this potential bias. A nine-point smoothing of the surface pressure field is applied before calculating the surface pressure gradients. The pressure gradients are then calculated using centered finite differences. For these forcings the skill of the wind-law (7) is compared to the skill that would be given by a model with no entrainment (MLM-N), as well as to the RFM and its counterpart the ARFM.

To choose the parameters for the respective models (except for one sensitivity study) we use those values that maximize the Murphree and van den Dool (1988) measure of joint-skill,

$$
\mathcal{S}=1-\frac{\sum\left[\left(U-U_{\text {coads }}\right)^{2}+\left(V-V_{\text {coads }}\right)^{2}\right]}{\sum\left(U_{\text {coads }}^{2}+V_{\text {coads }}^{2}\right)},
$$

where the summation is over the analysis domain. Maximizing $\mathcal{S}$ is equivalent to minimizing the standardized mean-square error. An alternative measure of skill, the mean-square correlation, $r$, of the two components of the wind, was also considered; and while results based on this measure were consistent with 
those presented here, it tended to be a less sensitive measure and thus was not chosen as the basis for the optimization.

Table 1: Skill, model parameters, and regression data for model winds versus COADS surface wind climatology for the Northern-Hemisphere winter season. Results from both the RFM and ARFM models are presented as well as three different implementations of the mixed-layer model MLM. MLM-N is the special case with no entrainment, MLM-O is the optimally fitted MLM. MLM-P illustrates the behavior of the MLM with perturbed, (i.e., non-optimal), parameter values. For each case the relevant parameter values are also listed. The regression fit data includes the regression coefficients $\left(m_{u}, m_{v}\right)$ and offsets $(u, v)$ as well as the mean-square correlation, $r=\sqrt{0.5\left(r_{u}^{2}+r_{v}^{2}\right)}$.

\begin{tabular}{lccccccccc}
\hline Wind-law & \multicolumn{3}{c}{ Skill } & \multicolumn{4}{c}{ Parameters } & \multicolumn{4}{c}{ Regression Fit Data } \\
& $\mathcal{S}$ & $\mathcal{S}_{u}$ & $\mathcal{S}_{v}$ & & $m_{u}$ & $m_{v}$ & $u$ & $v$ & $r$ \\
\hline \hline RFM & 0.72 & 0.83 & 0.89 & $\varepsilon=2.2 \times 10^{-5} \mathrm{~s}^{-1}$ & 1.31 & 1.07 & -0.96 & 0.24 & 0.82 \\
ARFM & 0.80 & 0.83 & 0.96 & $\left(\varepsilon_{x}, \varepsilon_{y}\right)=(1.6,4.2) \times 10^{-5} \mathrm{~s}^{-1}$ & 1.31 & 0.93 & -1.09 & 0.32 & 0.83 \\
\hline MLM-N & 0.73 & 0.86 & 0.87 & $h=338 \mathrm{~m}$ & 1.01 & 1.42 & -1.03 & 0.28 & 0.85 \\
MLM-O & 0.92 & 0.95 & 0.97 & $h=326 \mathrm{~m}, w_{e}=0.88 \mathrm{cms}^{-1}$ & 1.06 & 0.87 & -0.68 & 0.31 & 0.91 \\
MLM-P & 0.91 & 0.94 & 0.97 & $h=500 \mathrm{~m}, w_{e}=1.00 \mathrm{cms}^{-1}$ & 1.20 & 0.97 & -0.74 & 0.32 & 0.91
\end{tabular}

Table 1 summarizes the results of these comparisons. For the RFM models, the coefficients that maximize $\mathcal{S}$ are, in each case, consistent with previous analyses. Also in accord with previous studies, the skill of a model based on a Rayleigh drag increases significantly if this drag is allowed to depend on wind direction, and $\varepsilon_{y}$ is consistently two to three times larger than $\varepsilon_{x}$. Of the three MLM cases we consider, MLM-N is the optimal solution for no entrainment; MLM-O is the solution that maximizes $\mathcal{S}$; and MLM-P is a solution that shows the sensitivity of the MLM to its parameter values. The similarity in skill between the MLM-P and MLM-O solutions reflects the fact that the skill tends to rather flat along a line of increasing $w_{e}$ and $h$ in parameter space, which implies that the individual parameters are not as strongly selected as is their ratio. In fact the MLM-P solution is relatively closer to the parameter values that maximize $r$, thereby illustrating the extent of differences one might expect by optimizing around a different skill measure. Recall from our discussion of (7) in $\S 2$.a that MLM-N and RFM should be nearly equivalent: the only difference between the two models is that MLM still uses a quadratic drag. The results indicate that this leads to very slight, and probably negligible, improvements in the statistical measures considered.

Both the ARFM and MLM-O/MLM-P solutions have two free parameters that are selected through an optimization procedure, although if they exhibited commensurate skill one would still prefer the MLM because its parameters are more readily justified on physical grounds. However MLM-O is markedly more skillful than ARFM. This suggests that the coupling between $\mathbf{U}_{T}$ and $\mathbf{U}$ (which is included by way of the entrainment parameterization in the MLM, but neglected in Rayleigh Friction type models) is important and that simply allowing for anisotropy in the damping timescales for the wind does not properly incorporate this effect.

Using the MLM-O approach, we have repeated the above analysis for other seasons. There does not appear to be a pronounced seasonal dependence of optimal parameters. Overall ABL depths tend to be shallowest in northern winter and summer. In these seasons they are 326 and $365 \mathrm{~m}$, respectively, increasing to approximately $400 \mathrm{~m}$ in the other seasons. Entrainment rates vary between $0.9 \mathrm{cms}^{-1}$ and $1 \mathrm{cms}^{-1}$ with the larger entrainment rates in the seasons where the ABL is deeper. Our model actually is least skillful for the Boreal-winter season. Fits for other seasons result in marginally greater $(\mathcal{S}=0.93)$ skill. Overall, optimal ABL depths are somewhat shallower than we might have predicted; however, these should be interpreted as effective depths necessary for the bulk winds to reproduce the surface climatology (see $\S 4$ ). 

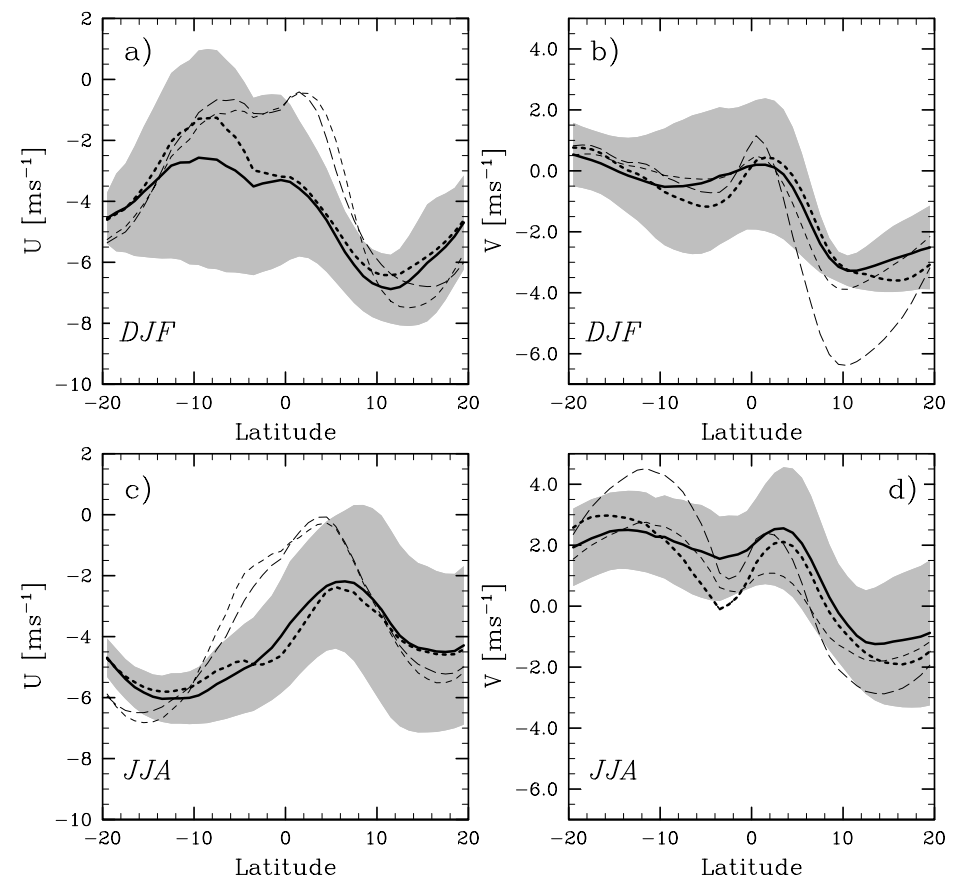

Figure 2: Zonally averaged winds for December-February (panels a \& b) and June-August (panels c \& d) forcing. Shown are the observations (bold-solid), and MLM-O (bold-dotted), ARFM (short-dash) and MLM-N (long-dash) solutions for the zonal (panels a \& c) and meridional (panels b \& d) components of the wind. Because of the similarity between MLM-O the RFM results from the latter are not shown. The shaded band has a width of two standard deviations about the mean and gives a rough measure of the variability of the COADS surface winds about their zonal means.

To the extent one is interested in $h$ and $w_{e}$, the MLM can be readily inverted to yield equations for these parameters as a function of the observed surface winds and the forcing. In principle one could then use these relations to make maps of the values of of $h$ and $w_{e}$ necessary for the MLM to exactly reproduce the surface wind climatology for the given forcing. It turns out that where this procedure produces values of $h$ and $w_{e}$ that are readily interpretable (i.e., greater than zero) the values are consistent with our previous analysis. However, because the maps also include broad regions where the model fails to be perfectly skillful for physically plausible parameter values, and because these regions tend to depend on which forcing data-set one uses, the exercise is less informative than one might initially suspect.

\section{2) PATtERnS}

A more concrete measure of the model skill is given in Fig. 2 where we compare zonally averaged winds derived from the MLM-O, MLM-N and ARFM to the December-February and June-August climatology. The superiority of the MLM-O model is also evident in the zonal means. As might be expected the improvement of the MLM-O winds relative to those predicted by the ARFM model is manifest mostly in the zonal flow near the equator. The improvement relative to the MLM-N solution is more global. In particular, the absence of entrainment not only leads to much too weak zonal flow at the equator, but also the equator-ward flow in the winter trades is too strong in the absence of entrainment. The chief shortcomings of the DJF MLM-O solutions appear to be that its prediction of the zonal flow near 10S is too weak and it predicts $\approx 1$ $\mathrm{ms}^{-1}$ northerlies at $5 \mathrm{~S}$ where the observed zonally averaged meridional flow tends to be closer to zero. The 
poor representation of the meridional flow at $5 \mathrm{~S}$ is even more evident in JJA (panel d).

The strengths and shortcomings of the MLM-O solutions can be further assessed by looking at the actual patterns of the wind relative to the climatology (Fig. 3). In this plot the DJF winds are displayed by vectors overlaying the divergence. Comparing panels (a) and (b) [or simply looking at the normalized differences in panel (c)] shows that the problems with the zonal flow near 10S are isolated in the far west Pacific, while the poor representation of the meridional flow near $5 \mathrm{~S}$ is most evident in the east Pacific. The poor representation in the west Pacific is in a region with very weak flow, vanishing pressure gradients, increasing continental influences, and perhaps less reliable data. Thus we hesitate to over-interpret the differences.

The biases in the east Pacific appear robust and are perhaps more interesting. Although the MLM-N solutions are worse than the MLM-O solutions almost everywhere, the one region where the MLM-N winds are superior is in the east Pacific near 5S. This result is evident in Fig. 2b as well as in wind vector plots of MLM-N (not shown). Consequently errors in the MLM-O solutions in the south-eastern Equatorial Pacific might largely reflect a failure to account for the spatial variation of entrainment, rather than the neglect of some other process. Indeed the generally accepted explanation for the acceleration of southerly flow over the equatorial cold tongue is that as air moves over colder water, the surface flow decouples from the flow aloft (Wallace et al., 1989), which implies less entrainment, thus leading to relatively more turning of the flow.

The above result suggests that one very tangible effect of entrainment is to retard the turning of the flow, leading to less convergence than might otherwise be expected. Maps of divergence (Fig. 3) are rather reasonable, thus supporting this idea. Most of the major features of the observations are evident, albeit with perhaps too much meridional structure. In contrast both the MLM-N and ARFM solutions (not shown) lead to substantially larger errors. These errors are both of greater amplitude and more zonally banded as both models tend to over-estimate equatorial convergence. This is especially true in the MLM-N representation of the winter trades, wherein the northerly flow is too pronounced. This leads to excessive convergence along a line just north of the equator which spans the east-west extent of the basin. These results suggest that, as long as the ABL momentum budget is consistently modeled, it may not be necessary to contrive other processes to account for the observed patterns of the wind.

\section{c. Entrainment}

The effect of entrainment on the force balance in our solutions is further explored through a consideration of various manifestations of this balance. Fig. 4 a illustrates the mean force balance for the entire winter tradewind region (which is rather steady and uniform; e.g., see Fig. 3) between 5 and $20 \mathrm{~N}$. The force balance is primarily an Ekman balance, with surface drag rotating the winds in the direction of lower pressure. As we discussed above, the primary effect of entrainment is to resist this rotation. In panel (b) the force balance is shown between $5 \mathrm{~S}$ and the equator for the entire basin. As suggested by Fig. 2a, in this region, and in the deep tropics in general, entrainment is much more important, so that the primary balance is between entrainment and surface drag. It is, however, worth noting that what we call entrainment in our model may compensate for the absence of other processes, such as non-linear advection or transients. If these were included the entrainment contribution to the force balance might be lessened.

The effect of entrainment can also lead to unexpected balances, such as illustrated in panel (c) which reflects the force balance near the dateline and 15S. Here entrainment dominates surface drag and leads to a rotation of the winds toward higher pressure. In this balance vertical mixing is working against the pressure gradient. To get such a balance requires that the forcings above the ABL be considerably different than those in the ABL, or that winds above the ABL be far from geostrophic. The former possibility implies considerable baroclinicity, which calls into question our use of the surface pressure gradient as a proxy for the mean ABL pressure gradient. Nonetheless the solutions remain physically plausible and it would be 


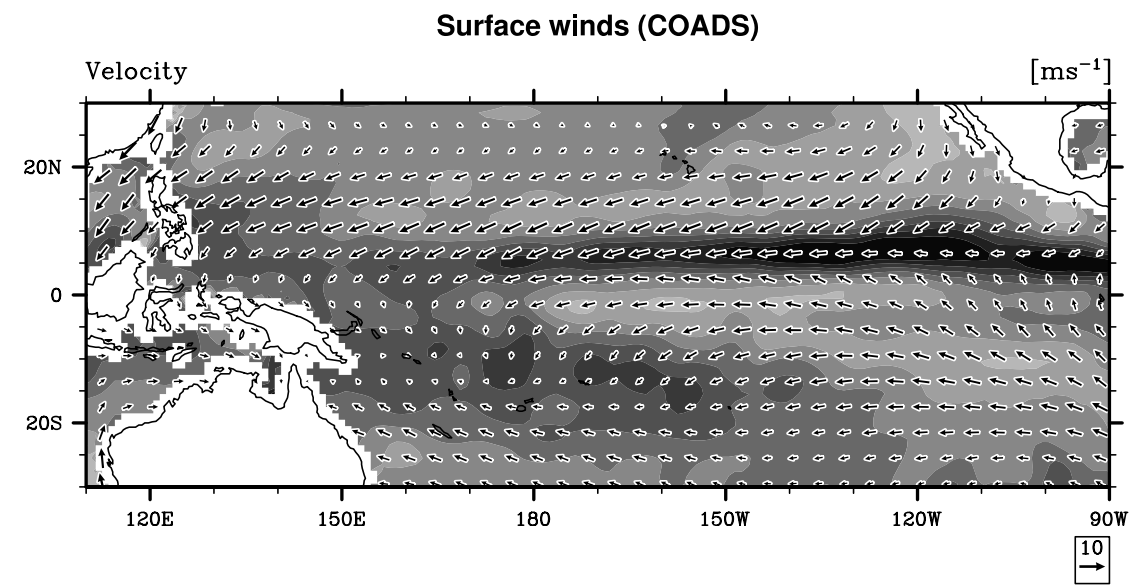

Surface winds (MLM-O)

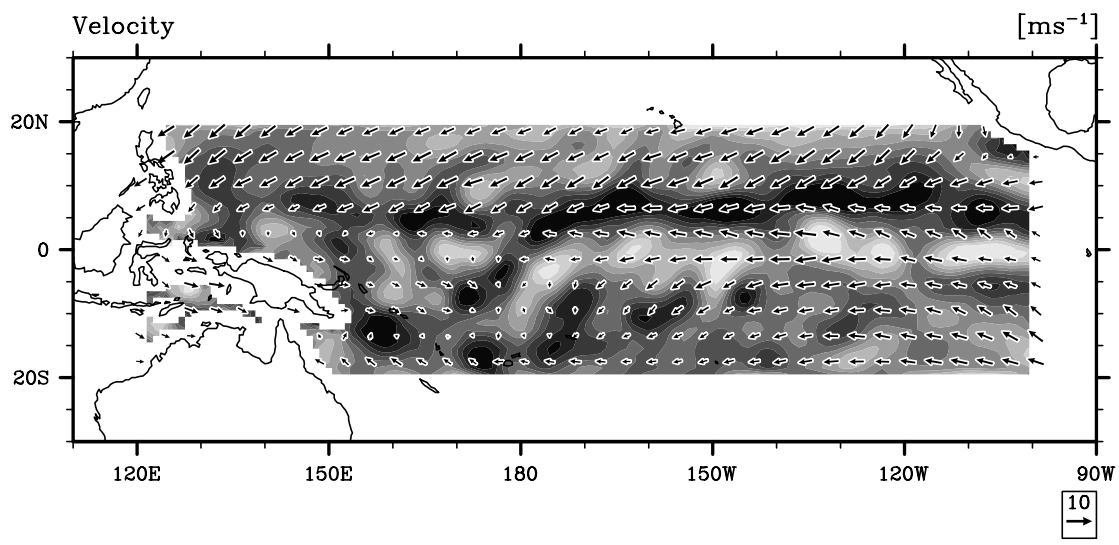

Surface winds (COADS - MLM-O)

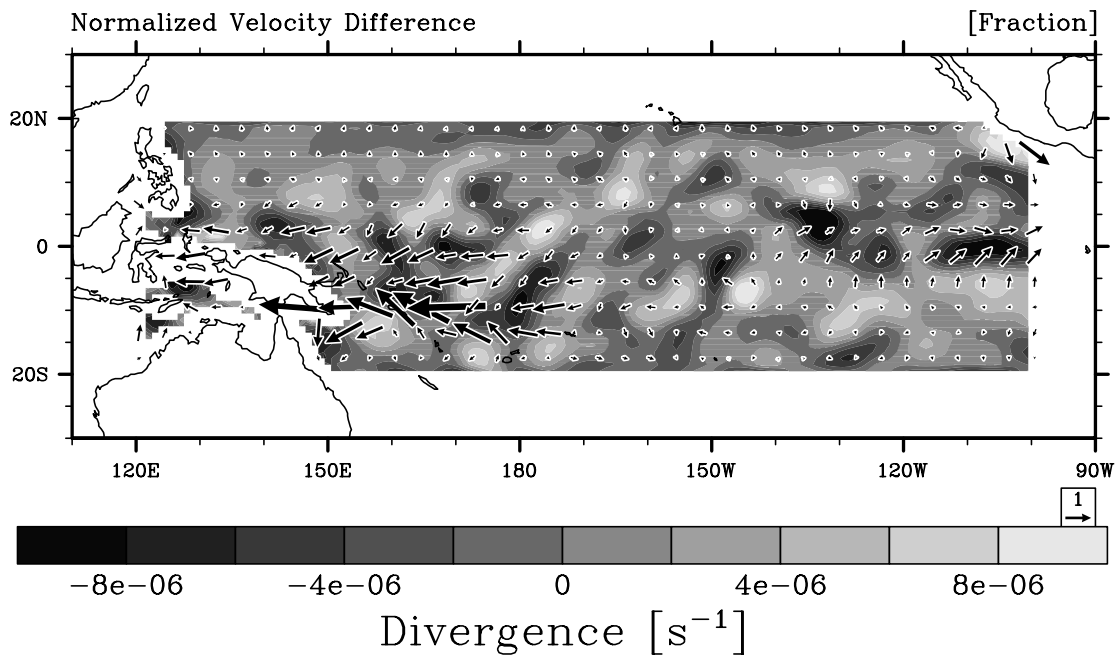

Figure 3: Vectors of wind overlaying contours of wind divergence: (a) COADS; (b) MLM-O solutions; (c) COADS MLM-O. Note that in panel (c) the differences are normalized by the magnitude of the observed winds, consequently the reference arrow length is unity here, rather than $10 \mathrm{~ms}^{-1}$. This method of displaying the errors tends to exaggerate the West Pacific errors where the winds are small. Note that panels (b) and (c) are plotted in a way that highlights the analysis domain. 


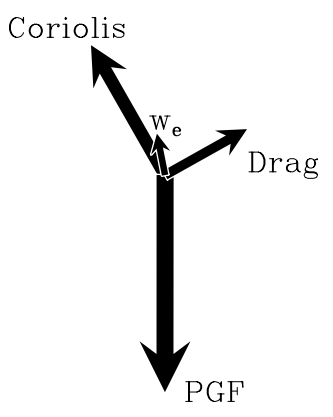

(a)

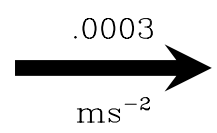

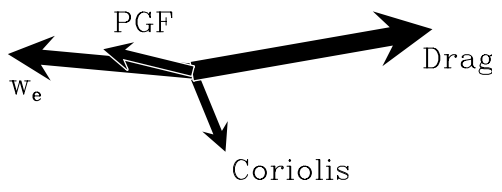

Coriolis

(b)

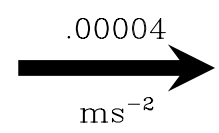

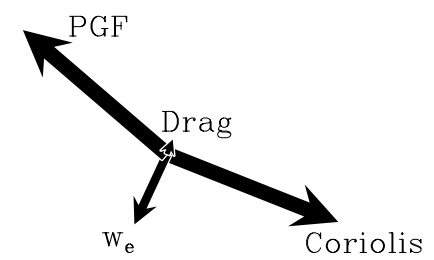

(c)

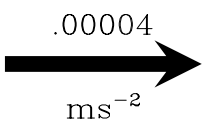

Figure 4: Vector averaged accelerations of the bulk wind for three different regions for December-February climatological data over the Pacific: (a) the region spanning $5-20^{\circ} \mathrm{N}$; (b) the region spanning $0-5^{\circ} \mathrm{S}$; (c) a single grid cell centered at $13.5^{\circ} \mathrm{S} 185.5^{\circ} \mathrm{E}$. Here PGF designates the pressure gradient force, drag designates surface drag, $w_{e}$ designates accelerations associated with entrainment, and Coriolis designates Coriolis accelerations. Note that because the drag is non-linear the mean wind is directed roughly (rather than exactly) opposite to the mean drag vector, and that the reference vector in panel (a) is nearly an order of magnitude larger than the reference vectors in the other panels.

interesting to see to what extent such anomalous balances play a role in climatological circulations.

To better quantify the role of entrainment over the whole tropical Pacific, we introduce an effective entrainment-stress velocity scale,

$$
u^{*}=\|\boldsymbol{\tau}(h)\|^{1 / 2},
$$

which is analogous to the surface friction velocity $u_{*}=\|\boldsymbol{\tau}(0)\|^{1 / 2}$. Because the entrainment velocity $w_{e}$ is really only an effective value (which in any optimization procedure will adjust to compensate for biases in the estimate of $\mathbf{U}_{T}-\mathbf{U}$ so as to produce the optimal flux) the scale velocity, $u^{*}$, is the more physically relevant measurement of entrainment. Denoting averages over the analysis domain by angle brackets, we note that for the MLM-O case $\left\langle u^{*}\right\rangle \approx 0.15$, which may be compared to $\left\langle u_{*}\right\rangle \approx 0.2$. If we consider only the region between $5 \mathrm{~N}$ and $5 \mathrm{~S},\left\langle u_{*}\right\rangle$ falls to about 0.17 and $u^{*}$ remains relatively unchanged. This result is not seasonally dependent, nor is it sensitive to the depth of the ABL. However, doubling the entrainment rate leads to approximately equal values of $u_{*}$ and $u^{*}$ for both the whole basin and the deep tropical region. This does not mean that entrainment and drag offset one another; from Fig. 4 it is evident that the entrainment and drag accelerations are often not aligned.

\section{d. Implied linear friction coefficients}

Given that the MLM satisfactorily represents the surface winds, can it be used to help understand and justify the ARFM? We proceed by first showing that the existing climatology of $\varepsilon_{x}$ and $\varepsilon_{y}$ is implicitly captured by the MLM. By deconstructing the MLM and its solutions we can show why this is. We do so not because we find $\varepsilon_{x}$ and $\varepsilon_{y}$ of particular physical interest, but rather because the peculiar behavior of $\varepsilon_{x}$ and $\varepsilon_{y}$ derived from fitting the ARFM to the observations has been the focus of so much previous analysis.

We first consider global estimates of $\varepsilon_{x}$ and $\varepsilon_{y}$ derived using the same procedure as Deser (1993), with the only difference being that we use the MLM derived winds in our analysis whereas she used the observations. In Table 2 our estimates of $\varepsilon_{x}$ and $\varepsilon_{y}$ are compared with those derived by Deser on the basis of the observed winds. Note that we use the MLM-P winds because we wanted to use seasonally constant 
Table 2: Regression estimates of $\varepsilon_{x}$ and $\varepsilon_{y}$ and wind-offsets diagnosed from MLM-P solutions $(h=500 \mathrm{~m}$ and $w_{e}=0.01 \mathrm{~ms}^{-1}$ ), and from Table 1a of Deser.

\begin{tabular}{lrrrrrrrr}
\hline Season & \multicolumn{2}{c}{$\varepsilon_{x}{ }^{-1}$ (days) } & \multicolumn{2}{c}{$\varepsilon_{y}{ }^{-1}$ (days) } & \multicolumn{2}{c}{$u_{o}\left(\mathrm{~ms}^{-1}\right)$} & \multicolumn{2}{c}{$v_{o}\left(\mathrm{~ms}^{-1}\right)$} \\
& MLM-P & Deser & MLM-P & Deser & MLM-P & Deser & MLM-P & Deser \\
\hline DJF & 0.79 & 0.74 & 0.33 & 0.27 & -0.7 & -0.7 & -0.1 & -0.1 \\
MAM & 0.56 & 0.69 & 0.32 & 0.23 & -1.8 & -1.1 & 0.0 & 0.1 \\
JJA & 0.59 & 0.61 & 0.35 & 0.24 & -1.7 & -1.4 & 0.2 & 0.5 \\
SON & 0.60 & 0.56 & 0.36 & 0.28 & -1.6 & -1.4 & 0.2 & 0.3
\end{tabular}

values of the parameters so as to isolate sources of seasonal variability in the results. We could have used parameters from the MLM-O solutions taken for any one of the seasons, but the MLM-P parameters take rounder values without changing the nature of the results. Overall the agreement is rather good. Not only does our wind-law produce similar regression estimates of $\varepsilon_{x}$ and $\varepsilon_{y}$ but the best-fit regression lines are also found to lie off the origin, with offsets commensurate to those found by Deser based on observed surface winds. A kind eye might even conclude that the model captures some of the observed seasonal variability.

In addition to reflecting the mean structure of the basin, the MLM solutions also imply similar meridional structure in the coefficients. In Fig. 5 we plot $\varepsilon_{x}$ and $\varepsilon_{y}$ as a function of latitude for December-February and June-August. Here calculate $\varepsilon_{x}$ and $\varepsilon_{y}$ by taking the average at each point in the latitude band, we note that the latitudinal dependence is quite similar to that derived by ( $\mathrm{Li}$ and Wang, 1994, cf., their Fig. 5), and that exhibited in the observations. This is most evident in the winter hemisphere. While $\varepsilon_{x}$ tends to increase uniformly away from the equator, $\varepsilon_{y}$ tends to have more of a " $U$ " shape. That is, $\varepsilon_{y}$ tends to increase more sharply with latitude in the near equatorial region, but less sharply away from the equator (this is most evident between 5-10 N/S in panel d). Similar features are also evident in the analysis of Chiang and Zebiak (2000).

These comparisons suggest that the improved skill of the constant-parameter MLM is commensurate to what one might arrive at through the use of the ARFM with latitudinally-varying coefficients. The entrainment effect seems to account for both the anisotropy and the latitudinal dependence in the friction coefficients derived from fits of (3) to the wind. Because our results are for globally fixed parameter values it does not appear to be necessary to argue for changes in $h$ with latitude (cf., Chiang and Zebiak, 2000) to explain the observed increase in $\varepsilon_{x}$ and $\varepsilon_{y}$ off of the equator.

Considerable insight into the behavior of $\varepsilon_{x}$ and $\varepsilon_{y}$ as implied by the MLM can actually be obtained directly from our wind-law. Separately considering the zonal and meridional components of (7), and assuming that their respective forcings satisfy ( $4 a$ ) and ( $4 b$ ), leads to the following expressions for $\varepsilon_{x}$ and $\varepsilon_{y}$ :

$$
\begin{aligned}
& \varepsilon_{x}=\frac{\tau_{x}(h)-\tau_{x}(0)}{h U}=\left(\frac{\|\mathbf{U}\| C_{D}+w_{e}}{h}\right)-\frac{w_{e}}{h}\left(\frac{U_{T}}{U}\right) \\
& \varepsilon_{y}=\frac{\tau_{y}(h)-\tau_{y}(0)}{h V}=\left(\frac{\|\mathbf{U}\| C_{D}+w_{e}}{h}\right)-\frac{w_{e}}{h}\left(\frac{V_{T}}{V}\right) .
\end{aligned}
$$

Here we see that the friction coefficients implied by the bulk-wind law consist of an isotropic and an anisotropic component, where the latter arises from the entrainment and is proportional to the ratio of the free-tropospheric component of the wind to value in the ABL.

Further analysis is aided by focusing on the specific flow regime wherein $\left\|U_{T}\right\| \gg\left\|V_{T}\right\|$, and $\|\partial P / \partial y\| \gg\|\partial P / \partial x\|$, chosen because of its similarity to the tropical climatology wherein zonal flow 

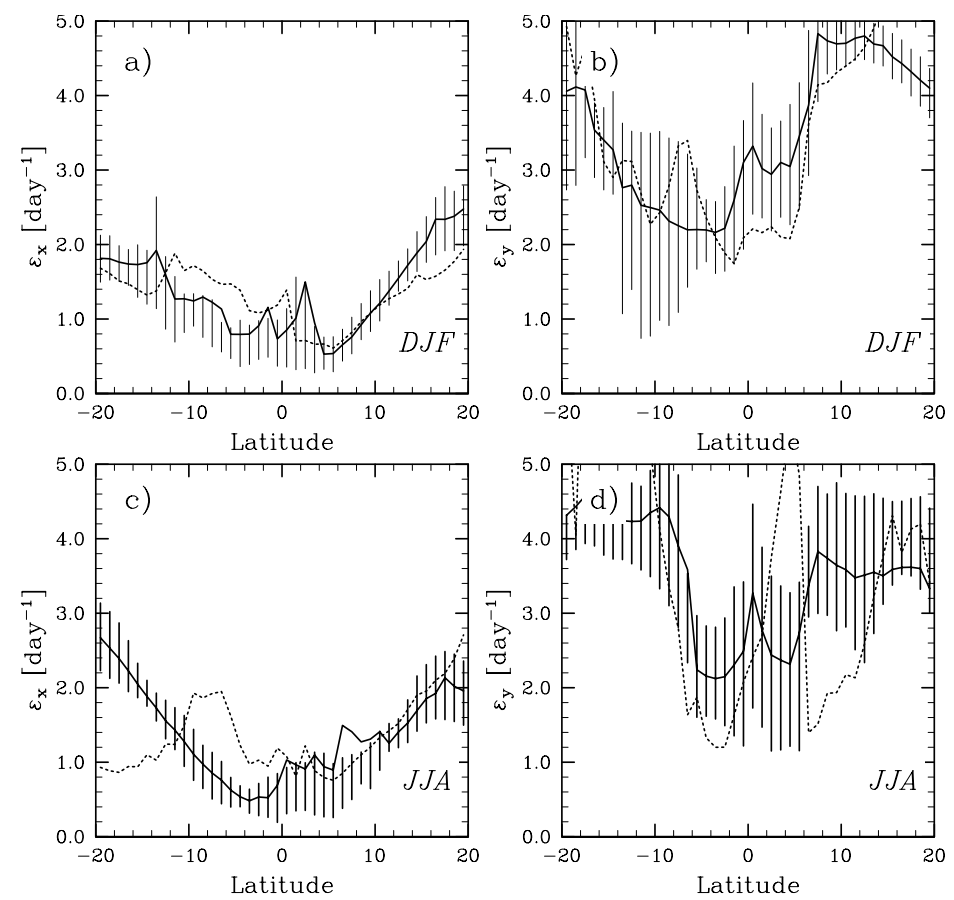

Figure 5: Friction coefficients for the ARFM as diagnosed from MLM-O solutions for December to February climatology (panels a \& b) and June to August (panels c \& d) for the tropical-Pacific: $(\mathrm{a}, \mathrm{c}) \varepsilon_{x} ;(\mathrm{b}, \mathrm{d}) \varepsilon_{y}$. The vertical bars delineate the range of values lying between the first and third quartile of the distribution, and the solid lines denote the mean. The dotted lines show the climatological values of $\varepsilon_{x}$ and $\varepsilon_{y}$ computed using observed surface winds. In computing the zonal means we exclude all points with negative coefficients or coefficients larger than 10 day $^{-1}$, this restriction is much weaker than that used by $\mathrm{Li}$ and Wang (1994) and eliminates between 10-15\% of the data although these exclusions are more concentrated in the near equatorial region. 
(particularly above the ABL) and meridional pressure gradients dominate. When we examine model solutions in this regime we generally find that $U_{T} / U>1$ and $V_{T} / V<1$, such behavior is also consistent with the ATEX wind profiles Brümmer et al. (1974), and reflects the basic Ekman tendency of the winds to turn toward lower pressure in the presence of friction. In terms of the two parameters

$$
\delta_{u}=\left(\frac{U_{T}}{U}-1\right), \quad \delta_{v}=\left(1-\frac{V_{T}}{V}\right),
$$

which denote the normalized departures of the bulk wind, we might expect $0<\delta_{u, v}<1$ in such circumstances. In this case the anisotropy of the friction coefficients, as measured by their ratio, becomes readily apparent,

$$
\frac{\varepsilon_{x}}{\varepsilon_{y}}=\frac{\|\mathbf{U}\| C_{D}-w_{e} \delta_{u}}{\|\mathbf{U}\| C_{D}+w_{e} \delta_{v}}<1
$$

Note that because the surface drag $\left(\tau_{x}(0)\right)$ must balance the entrainment momentum flux $\left(\tau_{x}(h)\right)$ in the absence of zonal pressure gradients at the equator $\lim _{f \rightarrow 0} \varepsilon_{x}=0$. Thus the tendency of $\varepsilon_{x}$ to increase away from the equator largely reflects a shifting balance as one moves off the equator - larger values of $\varepsilon_{x}$ reflect a diminishing role for entrainment.

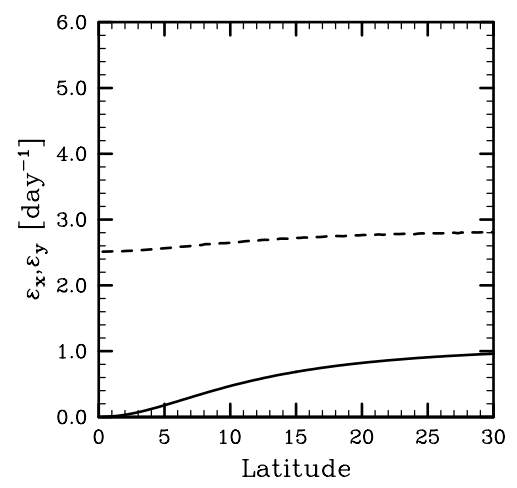

Figure 6: Friction coefficients for the ARFM as derived from the MLM for the baseline (solid lines) case in Fig. 1. Solid line $\varepsilon_{x}$, dashed line $\varepsilon_{y}$.

Fig. 5 can be more quantitatively interpreted by considering values of $\varepsilon_{x}$ and $\varepsilon_{y}$ implied by the idealized flow of Fig. 1. For such a simple forcing the friction coefficients (which we plot in Fig. 6) take the form

$$
\begin{aligned}
& \varepsilon_{x}=\frac{1}{h}\left(\|\mathbf{U}\| C_{D}-w_{e} \delta_{u}\right) \\
& \varepsilon_{y}=\frac{1}{h}\left(\|\mathbf{U}\| C_{D}+w_{e}\right) .
\end{aligned}
$$

Although the corresponding behavior of $\varepsilon_{x}$ and $\varepsilon_{y}$ in Fig. 5 is not exact, the idealized problem illustrates many important features of the friction coefficients. Most importantly Fig. 6 reproduces the observed phenomenology wherein $\varepsilon_{y}$ is two to three times larger than $\varepsilon_{x}$ and both tend to increase off the equator. Our idealized problem, and (13a)-(13b) in particular, also provides a basis for understanding how variations in $w_{e}$ and $h$ might affect implied values of $\varepsilon_{x}$ and $\varepsilon_{y}$. Because $\varepsilon_{y}$ is relatively constant in the idealized problem but drops sharply in the equatorial zone in Fig. 5 and because climatological estimates of $\varepsilon_{x}$ do not vanish near the equator the idealized flow perhaps best represents the situations poleward of 10S (in the trades). 
Presumably zonal pressure gradients and other deviations in the forcings contribute more meaningfully to the structure of $\varepsilon_{x}$ and $\varepsilon_{y}$ in the neighborhood of the equator.

In summary, the puzzling behavior in $\varepsilon_{x}$ and $\varepsilon_{y}$ that arises when the ARFM is fit to the observations is an artifact of its failure to properly incorporate entrainment in the ABL wind budget. Because entrainment acts to restore the ABL flow to the free-tropospheric flow on a specified timescale it projects anisotropies in the free-tropospheric winds onto the ABL winds. These anisotropies in turn lead to anisotropies in the ARFM friction coefficients. In addition a diminishing role for entrainment away from the equator imparts latitudinal structure on the ARFM derived friction coefficients.

\section{A linear bulk model}

Notwithstanding its greater skill, an inconvenience of the MLM (7) is its non-linearity. However, results presented in $\S 2 . b$ indicate that the source of this non-linearity, the quadratic formulation of the drag, is not a critical aspect of the model. Rather entrainment seems to be the important factor. This encourages the evaluation of a modified linear model that includes the entrainment effect, but in which the drag term is linearized.

We linearize the drag in analogy to the entrainment stress,

$$
\boldsymbol{\tau}(0)=w_{d} \mathbf{U}
$$

Substituting this into Eq. 2 leads to a linear bulk-wind rule,

$$
f \mathbf{k} \times \mathbf{U}+\frac{1}{\rho_{0}} \nabla P=\frac{w_{e} \mathbf{U}_{T}-\left(w_{e}+w_{d}\right) \mathbf{U}}{h},
$$

whose solutions are readily obtained as a function of the forcings,

$$
\begin{aligned}
U & =\frac{U_{T} \varepsilon_{i} \varepsilon_{e}-\frac{1}{\rho_{0}}\left(f \frac{\partial P}{\partial y}+\varepsilon_{i} \frac{\partial P}{\partial x}\right)}{\varepsilon_{i}^{2}+f^{2}} \\
V & =\frac{V_{T} \varepsilon_{i} \varepsilon_{e}+\frac{1}{\rho_{0}}\left(f \frac{\partial P}{\partial x}-\varepsilon_{i} \frac{\partial P}{\partial y}\right)}{\varepsilon_{i}^{2}+f^{2}} .
\end{aligned}
$$

To simplify notation, and make clear the independent parameters of the model, the above solutions are expressed in terms of the inverse timescales,

$$
\varepsilon_{i}=\frac{w_{e}+w_{d}}{h}, \quad \text { and } \quad \varepsilon_{e}=\frac{w_{e}}{h} .
$$

This linear model, which is closely related to similar models often used to study the thermodynamic evolution of mixed layers (e.g., Betts, 1983), turns out to be quite skillful. Its skill level $(\mathcal{S})$ is generally around 0.86 independent of season. This is compared to $\mathcal{S} \approx 0.92$ for the MLM-O model and 0.80 for the ARFM model. Solutions that optimize $\mathcal{S}$ also tend to have values of $r$ that are similar, if slightly larger than the ARFM model. Because the skill of the model is very flat along a line (or ridge) corresponding to $\varepsilon_{i}$ between $(3 / 2) \varepsilon_{e}$ and $2 \varepsilon_{e}$ in $\left(\varepsilon_{w}, \varepsilon_{e}\right)$ space, the optimal parameters are not strongly selected. As long as $w_{d}$ and $w_{e}$ are both $\mathcal{O}\left(1 \mathrm{cms}^{-1}\right)$ - with $w_{d}$ perhaps a bit smaller than $w_{e}$ - the model demonstrates considerable skill. On the other hand away from this ridge, the skill of the model decreases rather more rapidly than it does in the other models. 
The model (15) is analogous to the ARFM, in that contains two distinct relaxation timescales, but these relaxation timescales now incorporated into the equation-set in a physically realistic manner. The similarity between the two models can be formalized by noting that

$$
\begin{aligned}
& \varepsilon_{x}=\varepsilon_{i}-\varepsilon_{e}\left(\frac{U_{T}}{U}\right) \\
& \varepsilon_{y}=\varepsilon_{i}+\varepsilon_{e}\left(\frac{V_{T}}{V}\right) .
\end{aligned}
$$

These relations re-express the previous explanation of the nature and origin of the differences between $\varepsilon_{x}$ and $\varepsilon_{y}$.

\section{Boundary-layer vertical structure}

Our previous analysis is an attempt to explain the pattern of the surface winds with estimates of the bulk winds. The question naturally arises as to the relationship between these two quantities. Although the winds in the lower 500m are often observed to be relatively well mixed (Riehl et al., 1951; Brümmer et al., 1974), there are gradients, particularly near the surface, that might be expected to lead to $10-20 \%$ differences between the two quantities. If we account for such differences can we better represent the winds? Moreover, having shown that results of previous analyses can be explained by including entrainment in the budget of the bulk wind, the question remains as to how these explanations might be modified if we account for the detailed vertical structure of the wind. To answer this question we repeat the main elements of the previous analysis with a model that attempts to account for the vertical structure of the winds.

\section{a. The K-profile parameterization}

Instead of integrating over the vertical structure we consider solutions to (1) based on the $K$-profile parameterization (KPP, e.g., Lettau, 1950; Troen and Mahrt, 1986; Holtslag and Boville, 1993; Large et al., 1994; Stevens et al., 2000) for the flux $\tau$ :

$$
\boldsymbol{\tau}=K \frac{d \overline{\mathbf{u}}}{d z}, \quad \text { where } \quad K=w_{s} h\left[\frac{z}{h}\left(1-\frac{z}{h}\right)^{2}+c_{1}\right]
$$

The shape of the exchange coefficient, $K$, is standard, except for the addition of the small constant $c_{1}$ which has been added to help regularize the system at the boundaries. The dimensionality of $K$ is contained in the $w_{s} h$ prefactor, where $w_{s}$ is a turbulent scale velocity and $h$ is again the ABL depth. Matching to Monin-Obukhov similarity theory for $z / h \ll 1$ requires that

$$
w_{s}=\frac{\kappa u_{*}}{\phi_{m}(z / L)}
$$

near the surface. Here $\kappa$ is von Kármán's constant, and $\phi_{m}$ is the standard non-dimensional gradient function for momentum and is a function of the non-dimensional height $z / L$ where $L$ is the Obukhov length. In the neutral limit $\phi_{m}$ is unity, yielding a log-layer at the surface. Most of the tropics is characterized by positive buoyancy fluxes at the surface, in which case $\phi_{m}$ is less than unity, and $w_{s}$ should increase to the Deardorff (or convective) velocity scale $w_{*}$ away from the surface. For typical trade-wind conditions $w_{*}$ is about unity 
and $\phi_{m}$ at $10 \mathrm{~m}$ is about 0.75 . Given this model of the stress, the new (KPP) wind-law becomes

$$
\begin{aligned}
& \frac{d}{d z}\left(K \frac{d \bar{u}}{d z}\right)=\frac{1}{\rho_{0}}\left(\frac{\partial p}{\partial x}\right)-f \bar{v} \\
& \frac{d}{d z}\left(K \frac{d \bar{v}}{d z}\right)=\frac{1}{\rho_{0}}\left(\frac{\partial p}{\partial y}\right)+f \bar{u}
\end{aligned}
$$

Solutions are sought that obey this rule in the interior and which are consistent with flux conditions supplied by our drag-law and entrainment laws at the interfaces.

For consistency with the MLM we consider results using the same entrainment rule (e.g., based on the bulk, Eq. 6) as in the previous analysis, although the model is readily formulated to allow $w_{e}$ to depend on tropospheric interior profiles, surface stability, and $u_{*}$. To quantify the effect of near surface gradients, we do modify the surface flux rule, here we model the surface stress $\left(u_{*}^{2}\right)$ following surface-layer similarity:

$$
\frac{u\left(z_{1}\right)}{u_{*}}=\int_{z_{0}}^{z_{1}} \frac{\phi_{m}(z / L)}{\kappa z} d z=\frac{1}{\kappa}\left[\ln \left(z_{1} / z_{0}\right)+\Psi_{m}\left(z_{1} / L\right)\right] .
$$

Here $\Psi_{m}$ is the non-dimensional profile function (e.g., Garratt, 1992), $z_{0}$ is a roughness height which we take to be $0.0001 \mathrm{~m}$, and $z_{1}$ (where $h \gg z_{1} \gg z_{0}$ ) is a height in the surface layer which we take as $10 \mathrm{~m}$. For neutral conditions $\Psi_{m}=0$, for stable conditions it is order unity, and for unstable conditions (as in much of the trades; e.g., Stevens et al., 2001) $\Psi_{m} \approx-1$ is a reasonable value. Despite the apparent simplicity of the system, the integration procedure is complicated, particularly in so far as the interior profiles are matched to the boundaries. It is discussed in more detail in Appendix A.

\section{b. Surface Versus Bulk Winds}

Albeit somewhat idealized, the KPP model does provide a framework for answering our question as to how our results might be biased by interpreting observations of surface winds using a model of the bulk winds. The KPP model as posed above also provides a framework for investigating the effect of surface stability, which might be expected to regulate the relationship between bulk and surface winds.

We begin to address the above questions by first examining the behavior of the system for the base forcings used to produce the MLM results in Fig. 1. The results from the KPP model forced in this manner are shown in Fig. 7. Included in this figure are the resultant wind profiles, which indicate the asymmetry or anisotropy in the meridional versus zonal direction, as well as the effects of stability. Overall the behavior of the vertically averaged KPP winds is very similar to the behavior of the bulk winds derived from the MLM. Differences that arise reflect the fact that, to maintain the same surface drag, the KPP solutions must generate larger bulk wind-speeds. This implies less turning in the bulk. The net effect on the turning angle for the surface winds is, however, ambiguous as it also depends strongly on latitude and stability.

Overall the effects of stability are more pronounced, with increasing differentiation between the surface wind and the bulk winds with increasing stability. Most of the stability effects are related to changes in $w_{s}$, as for the cases considered the proportionality relation between $w_{s}$ and $u_{*}$ varies by a factor of 5 , whereas the effective drag coefficient (modified by changes to $\Psi_{m}$ ) varies only by a few percent. For the given forcings the absolute differentiation between the surface winds and the bulk winds is most pronounced for the zonal direction, thereby leading to larger discrepancies in the meridional friction coefficient ( $\varepsilon_{y}$ is proportional to the difference between the surface zonal wind and the geostrophic wind; e.g., see (3).

We would expect stability effects to play an even larger role had we incorporated them into the definition of the entrainment rate and the ABL depth. The ability of the KPP model to unify ABL profiles, depths, entrainment velocities, and surface-layer similarity theory under a relatively simple framework is part of 

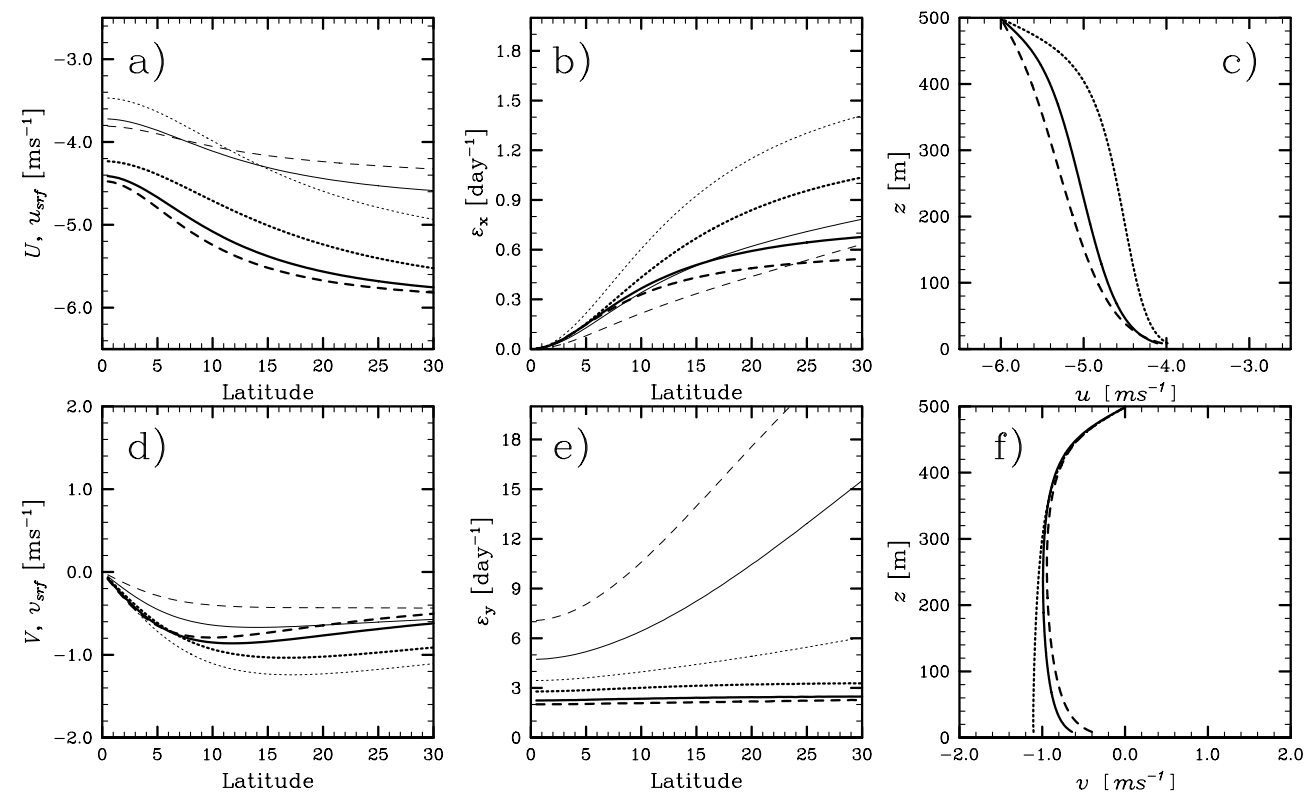

Figure 7: Results from KPP solutions for a neutral case (solid lines), an unstable ( $\Psi_{m}=-1.5, w_{s}=u_{*} / 0.3$ ) case (dashed lines), and a stable ( $\Psi_{m}=0.5, w_{s}=u_{*} / 1.5$ ), case (dotted lines); . Shown are: (a) bulk winds (thick lines), and surface winds (thin lines); (b) as in panel (a) but for diagnosed zonal friction coefficients, also note expanded scale relative to panel (b); (c) actual wind profiles. Panels (d)-(f) are identical to (a)-(c) except for meridional component of wind, note that the diagnosed $\varepsilon_{y}$ for the stable case are not shown because they are everywhere off the scale. The parameters $\left(h=500 \mathrm{~m}\right.$ and $\left.w_{e}=1 \mathrm{cms}^{-1}\right)$ are chosen to be consistent with the base case in Fig. 1 .

its appeal. The KPP model provides a convenient diagnostic framework for examining how a variety of boundary layer processes might contribute to the climatology of winds in the ABL. The fact that the parameterization is also widely used to model the winds prognostically in many large-scale models (e.g., Holtslag and Boville, 1993; Hong and Pan, 1996; Beljaars and Viterbo, 1998) makes its diagnostic application to climatology all the more appealing.

Lastly we investigate the KPP solutions for the entire Pacific basin. Because over most of this basin surface buoyancy fluxes are positive, we use parameter values corresponding to the unstable case. Overall the results from using this model do not differ substantially from our previous analysis with the MLM. Repeating the analysis of $\S 2$.b leads to similar skill $(\mathcal{S}=0.91$ for DJF). This skill value is actually marginally lower than that derived for case MLM-O $(\mathcal{S}=0.92)$. However, $\mathcal{S}$ is dependent on the stability parameters we use in the model, and no effort was made to fit these to the observations.

Overall the KPP model has skills commnesurate with the MLM, but for significantly deeper boundary layers with more entrainment. The ABL depth increases from less than 400m for the MLM to between 500 and $600 \mathrm{~m}$ for KPP. Similarly entrainment velocities increase from less than $1 \mathrm{cms}^{-1}$ for the MLM to about $1.5 \mathrm{cms}^{-1}$ for KPP. Although we do not have good observational estimates of $h$ and $w_{e}$ the optimal values from the KPP solutions are more in accord with our expectations. For instance, the sub-cloud layer in the trades is generally observed to be between 500 and 700m (Malkus, 1956; Brümmer et al., 1974, and many others). The rate of mixing across the sub-cloud layer is not readily measured in the atmosphere, although recent estimates on the basis of large-eddy simulation (Stevens et al., 2001), and the need for these mixing rates to balance the cumulus mass-flux across cloud-base on climatological timescales, suggest that values on the order of $2 \mathrm{cms}^{-1}$ are reasonable. Although here again we caution against over-interpreting values of $w_{e}$. It is the entrainment flux that is critical, and $w_{e}$ estimates might be compensating for biases in estimates 
of $\mathbf{U}_{T}$.

Armed with these insights we return to our question as to the relationship between our theory of the bulk winds, and observations of the surface winds. The KPP results suggest that interpreting the surface winds using bulk theory may bias ones estimates of $h$ and $w_{e}$ but such biases are modest, and have no qualitative impact on our discussions. Nonetheless, one should view the MLM derived values of $h$ and $w_{e}$ as effective, or equivalent, values necessary to optimize the correspondence between the bulk and the surface wind. If one is only interested in surface winds, it might be enough to use the mixed-layer framework, or the linearized variant thereof (with appropriately reduced values of $h$ and $w_{e}$ ). Such a model is however, likely to underestimate the bulk winds in the ABL and perhaps the mean convergence/divergence. To the extent such quantities are of interest the KPP derived solutions might be more appropriate. Although its computational complexity is considerably greater, KPP offers the advantage of providing a more unified view of $\mathrm{ABL}$ processes, thereby creating a framework within which one can better evaluate the role of a variety of processes in determining the surface wind climatology over the World Ocean.

\section{Concluding Remarks}

A bulk model of the winds appears able to represent well the seasonal climatology of surface winds over the tropical Pacific. The model demonstrates that entrainment, manifest as a turbulent stress at the boundary layer top, contributes at leading order to the ABL momentum budget. Including this process into a bulk budget of the winds considerably enhances the skill of the model. The effect of entrainment is particularly evident in the near equatorial region, as should be expected. Entrainment resists the tendency of the model to otherwise generate too much meridional flow in the trades. Consequently the incorporation of entrainment also leads to reasonable predictions of the divergence of the vector wind.

The key parameters of the model are the $\mathrm{ABL}$ depth $h$, and a mixing or entrainment rate $w_{e}$. The skill of the model can be optimized to yield best fit values for these parameters over the entire analysis domain. Such a procedure yields estimates of $h \approx 350-400 \mathrm{~m}$ and $w_{e} \approx 0.9 \mathrm{cms}^{-1}$, although given uncertainties in the data and the flatness of the skill, rounder values of $h=500 \mathrm{~m}$ and $w_{e}=1 \mathrm{cms}^{-1}$ are probably equally justified.

Overall the estimates of $h$ and $w_{e}$ derived by optimizing our bulk-wind law winds to those observed are smaller than we might have expected. Further analysis using steady-state solutions of the $K$-profile parameterization suggests that accounting for the vertical structure of the winds, rather than using the bulk wind as a proxy for the surface winds, leads to optimal values of $h$ and $w_{e}$ which are 25-50\% larger, and more in accord with our expectations. Although the $K$-profile solutions are not explored in great detail, the model does introduce further refinements into our approach which make it possible to more accurately assess the importance of what we think of as secondary processes, such as variations in surface stability, baroclinicity, and the variation of entrainment rates as a function of parameters (such as surface-layer stability) internal to the ABL. Its more general representation of the winds also provides a bridge between more elaborate theories of the surface winds and relatively simple ideas similar to those that we have focused on here.

The bulk model also illustrates why, when the momentum flux divergence is modeled as a Rayleigh drag, optimal damping coefficients are anisotropic. We show analytically that the shape of the winds within the ABL need not be accounted for to explain this result. Furthermore numerical results from the bulk model forced by climatology explain both the Deser (1993) finding that the best fit regressions of the winds to the left-hand side of (3) do not pass through the origin, as well as the observed latitudinal dependence of the regression, pointed out by Li and Wang (1994) among others.

Formally our bulk-wind law differs from the isotropic Rayleigh Friction model in two ways. First the drag is a quadratic rather than a linear function of the wind speed, and second entrainment is not neglected. The first modification makes the model non-linear, but the second is more important. This realization mo- 
tivates the introduction of a modified bulk-wind rule, which incorporates entrainment but is based on a linearized surface drag. The resultant two parameter model is linear, isotropic in its parameters (although not in its implied drag) and markedly more skillful than both the isotropic and anisotropic Rayleigh friction models.

Acknowledgments. This work was supported by kind assistance from the National Science Foundation, Grant \# ATM-9985413, and NOAA, Grant \#NA86GP0361. The NCEP Reanalysis data was provided by the NOAA-CIRES Climate Diagnostics Center, Boulder, Colorado, USA, from their Web site at http://www.cdc.noaa.gov/. ECMWF is thanked for making their data available through NCAR, as are all those who have contributed to the COADS. The first author thanks C.S. Bretherton for many stimulating discussions on this general topic and in particular his insights on the physical interpretation of entrainment and its possible relation to cumulus momentum fluxes. David Muraki is also thanked for fruitful conversations regarding the formulation of the solution method for the KPP model. The NCL group at NCAR (E. Albert, M. Haley, S. Murphy, D. Shea and others) are also thanked for providing a first-rate community analysis/graphics package. Lastly three anonymous reviewers are thanked for their constructive comments. 


\section{Appendix A \\ Solutions for the KPP model}

The system $(21 a)-(21 b)$ is integrated between two points, $z_{1}=10 \mathrm{~m}$ and

$$
z_{2}=h_{-}=\lim _{\epsilon-\rightarrow 0}(h-\epsilon)
$$

near its bounding surfaces. Because we match to flux boundary conditions at both surfaces, surface values of $\overline{\mathbf{u}}$ are not constrained, nor are gradients at either boundary. Instead the constraints on the system are that the winds at $h_{-}$match those above the $\mathrm{ABL},{ }^{2}$ and that the integral force budget is balanced. The latter consists of two integral constraints on the system,

$$
\begin{aligned}
& u_{*}^{2}\left[\frac{u\left(z_{1}\right)}{\left\|\mathbf{u}\left(z_{1}\right)\right\|}\right]=-\left[\frac{z_{2}-z_{1}}{\rho_{0}}\left(\frac{\partial P}{\partial x}\right)-\int_{z_{1}}^{z_{2}} f v(z) d z\right]+w_{e}\left(U_{T}-\frac{1}{z_{2}-z_{1}} \int_{z_{1}}^{z_{2}} u(z) d z\right) \\
& u_{*}^{2}\left[\frac{v\left(z_{1}\right)}{\left\|\mathbf{u}\left(z_{1}\right)\right\|}\right]=-\left[\frac{z_{2}-z_{1}}{\rho_{0}}\left(\frac{\partial P}{\partial y}\right)+\int_{z_{1}}^{z_{2}} f u(z) d z\right]+w_{e}\left(V_{T}-\frac{1}{z_{2}-z_{1}} \int_{z_{1}}^{z_{2}} v(z) d z\right) .
\end{aligned}
$$

The surface and entrainment fluxes only enter the solution here, through these constraints.

This leads to a generalized boundary value problem that we solve using a shooting method. By guessing values of $d \overline{\mathbf{u}} / d z$ at $z=z_{2}$ we integrate the model to $z_{1}$ and calculate the surface flux using (22), and the entrainment flux based on the mean trial-wind and our entrainment rule. Our guesses for $d \overline{\mathbf{u}} / d z$ at $z_{2}$ are then adjusted to eliminate any imbalance in the integral force balance $(A 1 a)-(A 1 b)$. Thus we shoot on the two component integral constraints for the system.

Because the mixing coefficient $K$ depends on the surface stress (through $u_{*}$ ) the above procedure actually yields a family of solutions for different values of $K$. We select the solution with the $K$ that is consistent with the implied value of $u_{*}$. That is in our shooting method we adjust our guesses so that we attempt to minimize not only force imbalances, but also differences between the value of $u_{*}$ used in the specification of $K$ and that implied by winds at the surface. Some limitations of our present framework are that the interior flux and the entrainment flux need not match. This reflects the fact that we do not trust the $K$-profile in the vicinity of the upper boundary. Also, because $K$ vanishes quadratically as $z \rightarrow h$ profiles become singular (see Stevens et al., 2000, for a discussion of this topic). The small constant $c_{1}$ is added to $K$ as a way around this problem (see Eq. 19). This constant affects the details of the profile near the upper boundary but not the nature of the overall solution, which in part supports our lack of concern that the $K$-profile and the entrainment flux match at $z=h$.

We use a fourth-order Runge-Kutta method for integrating the wind-law. Guesses are adjusted using Newton's method. Once convergence begins it is very rapid, but for many forcings not all initial conditions lead to convergence, thus at some points the solution procedure can be quite slow as we search for better starting points.

\footnotetext{
${ }^{2}$ Note that such a matching condition is not the only possible choice. Mixed layer models of the winds do not match the winds at the upper boundary, rather the fluxes. In the KPP solutions we could have instead chosen such an approach whereby the entrainment flux is matched to the interior flux at $h_{-}$.
} 


\section{References}

Augstein, E., H. Schmidt and F. Ostapoff, 1974: The vertical structure of the atmopheric planetary boundary layer in undisturbed trade winds over the Atlantic Ocean. Bound.-Layer Meteor., 6, 129-150.

Beljaars, A. and P. Viterbo, 1998: Clear and Cloudy Boundary Layers. chapter Role of the boundary layer in a numerical weather prediction model. Elsevier-North Holland, Amsterdam, A. A. M. Holtslag and P. G. Duynkerke, Eds.

Betts, A. K., 1983: Thermodynamics of mixed stratocumulus layers: saturation point budgets. J. Atmos. Sci., 40, 2655-2670.

Brümmer, B., E. Augstein and H. Riehl, 1974: On the low-level wind structure in the atlantic trade. Quart. J. Roy. Meteor. Soc., 100, 109-121.

Chiang, J. C. H. and S. E. Zebiak, 2000: Surface wind over tropical oceans: diagnosis of the momentum balance, and modeling the linear friction coefficient. J. Climate, 13, 1733-1747.

Da Silva, A. M., C. C. Young and S. Levitus, 1994: Atlas of surface marine data 1994 vol. 1, algorithms and procedures. Technical report, U. S. Department of Commerce, National Oceanic and Atmopsheric Administration, $83 \mathrm{pp}$.

Deardorff, J. W., 1972: Parameterization of theplanetary boundary layer for use in general circulation models. Mon. Wea. Rev., 100, 93-106.

Deser, C., 1993: Diagnosis of the surface momentum balance over the tropical Pacific ocean. J. Climate, 6 , 64-74.

Garratt, J. R., 1992: The atmospheric boundary layer. Cambridge, 372 pp.

Holtslag, A. A. M. and B. A. Boville, 1993: Local versus nonlocal boundary-layer diffusion in a global climate model. J. Climate, 6, 1825-1842.

Hong, S.-Y. and H.-L. Pan, 1996: Nonlocal boundary layer vertical diffusion in a medium-range forecast model. Mon. Wea. Rev., 124, 2322-2339.

Kalnay, E. and Coauthors, 1996: The NCEP/NCAR 40-year reanalysis project. Bull. Amer. Meteor. Soc., 77, 437-471.

Large, W. G., J. C. McWilliams and S. C. Doney, 1994: Oceanic vertical mixing: a review and a model with a nonlocal boundary layer parameterization. Reviews of Geophysics, 32, 363-403.

Lettau, H., 1950: A re-examination of the "Leipzig Wind Profile" considering some relations between wind and turbulence in the frictional layer. Tellus, 2, 125-129.

Li, T. and B. Wang, 1994: A thermodynamic equilibrium climate model for monthly mean surface winds and precipitation over the tropical Pacific. J. Atmos. Sci., 51, 1372-1385.

Lilly, D. K., 1968: Models of cloud topped mixed layers under a strong inversion. Quart. J. Roy. Meteor. Soc., 94, 292-309.

Lindzen, R. S. and S. Nigam, 1987: On the role of sea surface temperature gradients in forcing low-level winds and convergence in the tropics. J. Atmos. Sci., 44, 2440-2458. 
Malkus, J. S., 1956: On the maintenance of the trade winds. Tellus, 8, 335-350.

Matsuno, T., 1966: Quasi-geostrophic motions in the equatorial area. J. Meteor. Soc. Japan, 44, 25-42.

Murphree, T. and H. van den Dool, 1988: Calculating winds from time mean sea level pressure fields. $J$. Atmos. Sci., 45, 3269-3281.

Neelin, J. D., 1988: A simple model for surface stress and low-level flow in the tropical atmosphere driven by prescribed heating. Quart. J. Roy. Meteor. Soc., 114, 747-770.

Riehl, H., C. Yeh, J. S. Malkus and N. E. LaSeur, 1951: The North-East Trade of the Pacific Ocean. Quart. J. Roy. Meteor. Soc., 77, 598-626.

Stevens, B., A. A. Ackerman, B. A. Albrecht, A. R. Brown, A. Chlond, J. Cuxart, P. G. Duynkerke, D. C. Lewellen, M. K. MacVean, R. Neggers, E. Sanchez, A. P. Siebesma and D. E. Stevens, 2001: Simulations of trade-wind cumuli under a strong inversion. J. Atmos. Sci., 58, 1870-1891.

Stevens, B., C.-H. Moeng and P. P. Sullivan, 2000: Entrainment and subgrid lengthscales in large-eddy simulations of atmospheric boundary layer flows. in R. Kerr and Y. Kimura, editors, IUTAM Symposium on Developments in Geophysical Turbulence, pp. 253-269. Kluwer, Dordrecht.

Troen, I. B. and L. Mahrt, 1986: A simple model of the atmospheric boundary layer: sensitivity to surface evaporation. Bound.-Layer Meteor., 36, 129-148.

Wallace, J. M., T. P. Mitchell and C. Deser, 1989: The influence of sea-surface temperature on surface wind in the eastern equatorial pacific: seasonal and interannual variability. J. Climate, 2, 1492-1499. 